Confocal Microscopy System

Performance: Laser Power Measurements

Robert M. Zucker, PhD

U.S. Environmental Protection Agency, ORD, NHEERL zucker.robert@epa.gov

The reliability of the confocal laser-scanning microscope (CLSM) to obtain intensity measurements and quantify fluorescence data is dependent on using a correctly aligned machine that contains a stable laser power. The laser power test appears to be one of the most useful tests to quickly evaluate if a system is misaligned or functioning sub optimally by recording insufficient laser power readings. The test using a power meter can indicate if the system is aligned properly up to the plane of excitation on the stage, or if the machine has a defective component (i.e. a dying laser, or a defective fiber). In our experience, without sufficient power throughput in the system, the PMT voltages will have to be increased to high values to visualize fluorescence derived from specimens, which will introduce reduced image quality. In addition the cause of the decreased laser power may result in other problems i.e. laser instability, loss of axial resolution, and increased noise.

The equipment used to acquire power readings includes a detector, a machine shop built detector holder and a portable power meter (Figure 1). The power meter used to measure light on the

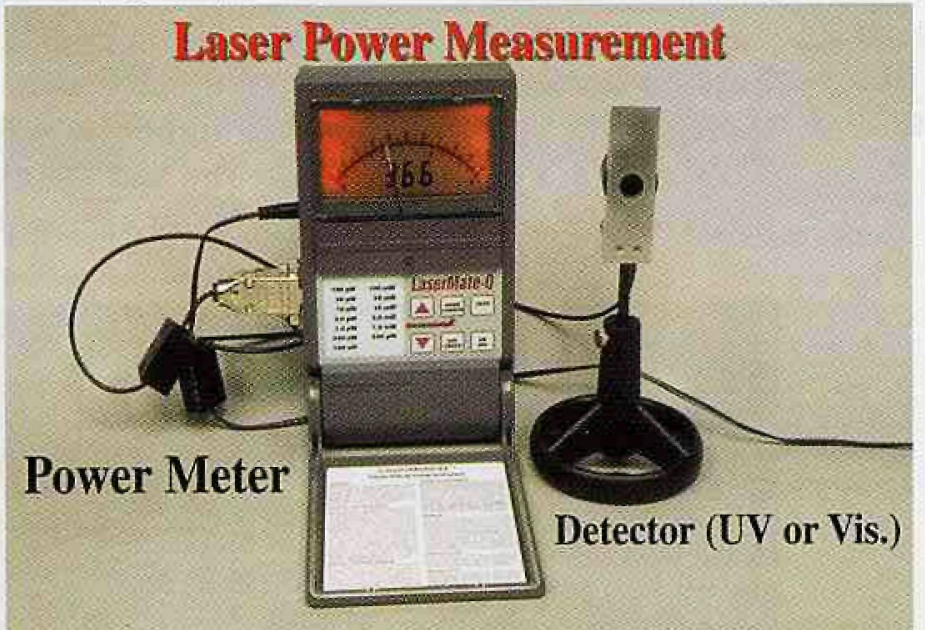

Figure 1. Equipment. The equipment used to acquire power readings include a detector, a machine shop built detector holder and a portable power meter.

microscope stage was a Lasermate $Q$ (Coherent, Auburn, California) with visible (LN36) and UV detectors ( $L 818$ ). The detector was secured in a special holder that fits onto the microscope stage in place of a $3 \times 1$ slide (Figure 2). A power meter (1830C) from Newport Corporation with an SL 818 visible wand detector (Figure 2) is a good alternative.

The test should be done with a dry objective (2.5x-20x, preferably $10 x$ ) at a fixed position, usually at the top of its moveable tract. On most confocal systems there is a $10 \mathrm{x}$ lens: Zeiss uses a $10 \mathrm{x}$ Plan Neofluar (NA 0.3) and a Leica has a 10x Plan Fluorotar (NA 0.3 ) or $10 \times$ Plan Apo (NA 0.4). The use of a lens that has a different design, magnification or NA will affect the laser power transmission and measurement. The test was made in the following manner: The $10 x$ objective is raised to its maximum specified height. The detector is secured on the stage and grossly centered using either
Coherent stage holder Newport stage holder

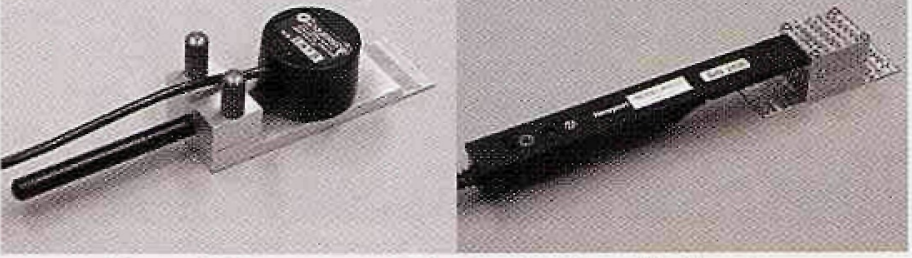

Figure 2-Power meter detector holders. Holders to secure detectors on the microscope stage were made in the machine shop in a size similar to a $3 \times 1$ microscope slide. The holders for the Coherent (LN 36) or Newport wand (SL818) detectors are represented in the photographs. The machinist's plans for building the power detector holder are available by e-mailing the author.

laser light or mercury fluorescent light. The detector position is then more accurately adjusted to achieve maximum signal intensity by using the microscope's x/y joystick. The power meter is adjusted to the specific wavelength $(365,488,568$, or $647 \mathrm{~nm})$ and the maximum power of laser light is read on the digital scale. The CLSM zoom factor is set from $8-32$ to reduce the beam scan and to focus it into the "sweet spot" of the detector. The scanner is set at bi-directional-slow speed to reduce the time period that the power meter is reading " 0 ". The maximum digital reading from the power meter was recorded. The power derived from this measurement is dependent on the type magnification and NA of the lens used. The power meter diode was not reliable and could only be used as a crude estimate of laser power. The ability of the built-in diode to measure power may occur in the future with newer designed CLSM systems.

A comparison of the maximum power output derived from different lasers, and different optical systems were made on a Zeiss 510, a Leica TCS-SP1 and a Leica TCS-SP2. Using a triple dichroic to reflect different wavelengths of laser light to the stage (table 1). In a three-laser system, there is sufficient power with the 633 and 488 lasers. Since the maximum power of the 543 laser is around $1 \mathrm{~mW}$, excessive attenuation of the 543-laser light may reduce the laser power to insufficient quantities (less than $0.2 \mathrm{~mW}$ ) that can produce unacceptable images. With insufficient power, the PMTs will need to be operated at high voltage settings, which increase the system noise. Low power throughput in the confocal system suggests a problematic laser, a fiber polarization problem or just a badly aligned system.

These values (Table 1) are useful to illustrate the maximum power that can be obtained from a CLSM having these different laser configurations. These measurements serve as a valuable reference for an individual lab to internally control an individual machine and they serve as a comparison to other similar CLSM systems. This power output test can help determine if the system is misaligned, functioning badly, or if the laser needs to be replaced. It should be emphasized that this test is performed at the microscope stage prior to the light reflecting the dichroics for a second time and penetrating through the emission pinhole and the emission barrier 


\section{DON'T OPERATE}

BLINDFOLDED

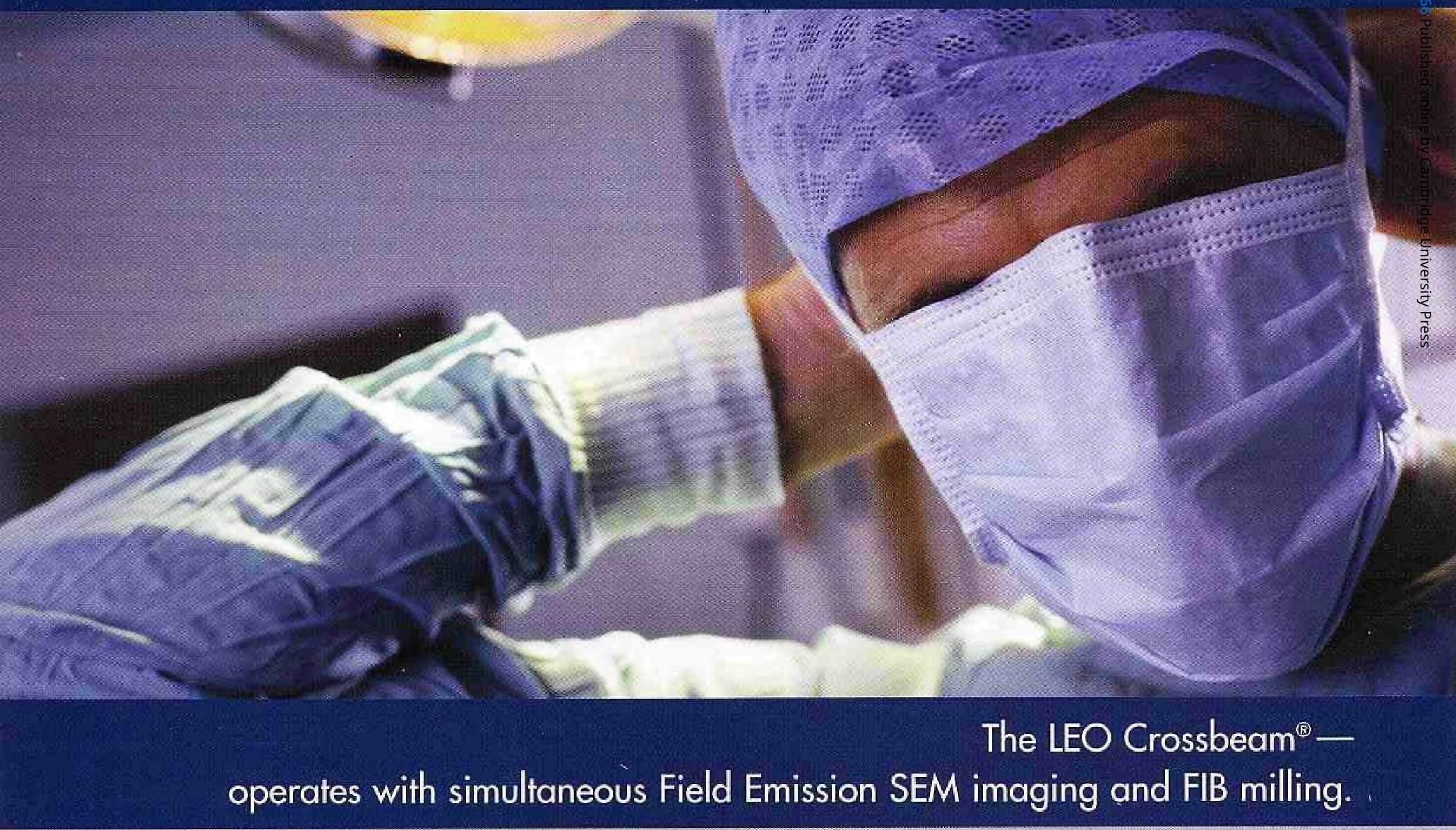

Only wh the LEO Grosibsam can you see your semple as you re miling it -

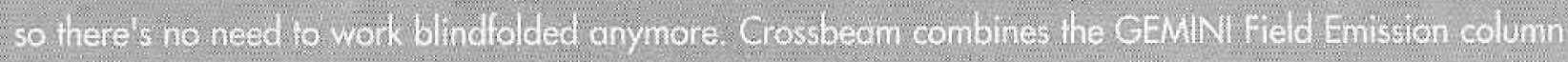

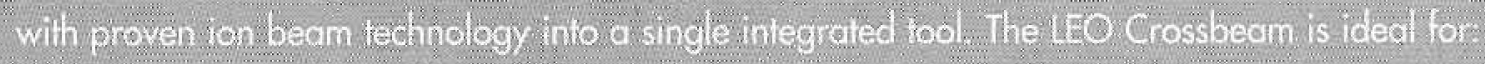

- Failure analysis

- TEM sample preparation

- Cross section investigation

- Three dimensional structural examination

Use the LEO Crossbeam and stop working blindfolded! Call 1-800-356-1090 today!

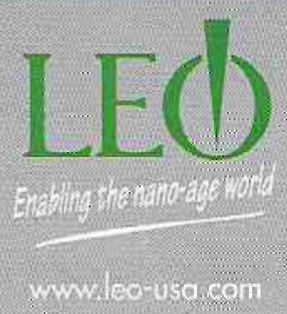




\begin{tabular}{|c|c|c|c|}
\hline \multicolumn{4}{|c|}{ Maximum Power Comparison } \\
\hline & Leica SP1 & Leica SP2 & Zeiss 510 \\
\hline Laser & Ar-Krypton & (3-laser) & (3-laser) \\
\hline $488 \mathrm{~nm}$ & 1.1 & 4.5 & 3.13 \\
\hline $543-568 \mathrm{~nm}$ & 1.45 & 0.22 & 0.26 \\
\hline $633-647 \mathrm{~nm}$ & 1.6 & 2.8 & 1.21 \\
\hline
\end{tabular}

Table 1. Comparison of Relative Power on Microscope Stage. The maximum power was measured in $m W$ with a $L N$ 36 detector and a Coherent Lasermate power meter adjusted to the specific excitation wavelengths. The power was measured on the stage of a Zeiss 510, a Leica SP2, and a Leica SP1 system. The maximum power was measured with a Coherent power meter using two different 10x (0.3NA) lenses on two different types of CLSM systems. The Leica TCS-SP1 (NA 0.3) system has one 75mW argon-krypton laser (model 643) emitting three laser wavelength lines. The newer CLSM systems from all manufacturers are designed with three lasers that use different dichroic components to merge the different laser wavelengths. The Zeiss 510 (NA 0.3) contained 3 different lasers ( $30 \mathrm{~mW}$ Argon $1 \mathrm{~mW}$, HeNe $(543 \mathrm{~mm})$, and a $5 \mathrm{mw}$ HeNe $(633 \mathrm{~nm})$ with a merge module. The Leica SP2 (NA0.4) contained 3 different lasers (50 $\mathrm{mW}$ Argon, $1.2 \mathrm{~mW}$ HeNe $(543 \mathrm{~nm}$ ), and a $10 \mathrm{mw}$ HeNe $(633 \mathrm{~nm})$ aligned with a merge module.

filters or prisms (if they exists in the system) and into the PMT. The values reported in Table 1 were acquired from functioning systems that were subjectively assessed to be problem free, aligned correctly and functioning properly. There are no assurances that these machines were producing ideal power readings or were perfectly aligned when these tests were made.

\section{UV power test}

The test was carried out in a similar manner to that described for the visible power measurement. A power meter (Lasermate/Q with UV detector (L818), Coherent Santa Clara California) was used to measure the light emitted from UV Enterprise laser. A Coherent UV, 60-mW, 3-year-old Enterprise laser delivered normal power output at the laser head (over $40 \mathrm{~mW}$ of laser power), but only about 500 $\mu$ watt maximum power through a Plan Fluor $10 \times(0.3 \mathrm{NA})$. However, it should be noted that when our system had insufficient output under these conditions (approximately $500 \mu$ watt through a $10 x$ Leica (0.3NA) lens) we also had insufficient light for many UV experiments using higher magnification objectives $(40 x, 63 x$, and $100 x)$. The UV power output was controlled using a remote control box to regulate UV laser power (0163-662-00, Coherent, Santa Clara, California).

\section{Bead or histological power meter}

Due to optical limitations of the stage, the power meter detector cannot be used with higher power lenses (40x,63x, 100x). Thus it will be useful to use a fluorescence histological slide (FluorCells slide, $F$ 14780, Molecular Probes, Eugene, Oregon) fluorescence colored plastic slides (Applied Precision), or bead sample to assess UV power with these higher power lenses. Using maximum UV power, the 10 micron Spherotech bead (Libertyville IL, FPS-10057-100) saturates PMT 1 (low noise PMT) at a voltage setting of 650 using a 100x Plan Apo lens (NA 1.4). Higher values of saturation indicate less power throughput in the CLSM system and lower powers of saturation indicate greater power throughput. The saturation values on other substrates or slides (biological or histological specimens) can also be used and have to be defined by the user's laboratory as the degree of staining can be different. In our opinion, these tests are very crude and subjective due to the acceptability of such a large range of PMT amplification values, the variations in staining between different plant samples, variations

in PMT characteristics and the logarithmic relationship between PMT vs. intensity (1). However, it does yield a rough estimate of whether there is sufficient power in the system.

If a power meter is not available, the crude power of the system can be assessed by recording the PMT voltage necessary to acquire an image at almost saturation values by using standard histological samples or 10 -micron Spherotech beads. If conditions are identical between machines, this PMT value can be used as a reference value to compare CLSM units and to establish their acceptable performance levels. Leica technicians routinely use a $40 x$ lens to measure the fluorescence saturation of a histological plant sample. If it saturates in the PMT range between 600-700 units in PMT 1 the system is passed as having adequate power. Scientists desiring a more accurate method to test performance will find problems with this type of testing due to the large range of PMT voltage values that can be considered acceptable.

\section{References:}

1. Zucker, R.M. Price OT Evaluation of confocal system performance. Cytometry 44:273-294 2001.

2. Zucker, R.M. Price OT Statistical evaluation of confocal microscopy images. Cytometry 44:295-308 2001

3. Carter D: Practical considerations for collecting confocal images. Methods Mol Biol. Confocal Microscopy Methods and Protocols edited by S. Paddock Humana Press, Totowa NJ. 122:35-57 1999.

1 The research described in this article has been reviewed and approved for publication as an EPA document. Approval does not necessarily signify that the contents reflects the views and policies of the Agency, nor does mention of trade names or commercial products constitute endorsement or recommendation for use.

${ }^{2}$ To whom all correspondence and reprint requests should be addressed: U.S. Environmental Protection Agency, Reproductive Toxicology Division (MD-72), National Health and Environmental Effects Research Laboratory, Research Triangle Park, NC 27711; Phone: (919) 541-1585; FAX: (919) 541-4017 e-mail:zucker.robert@.epa.go

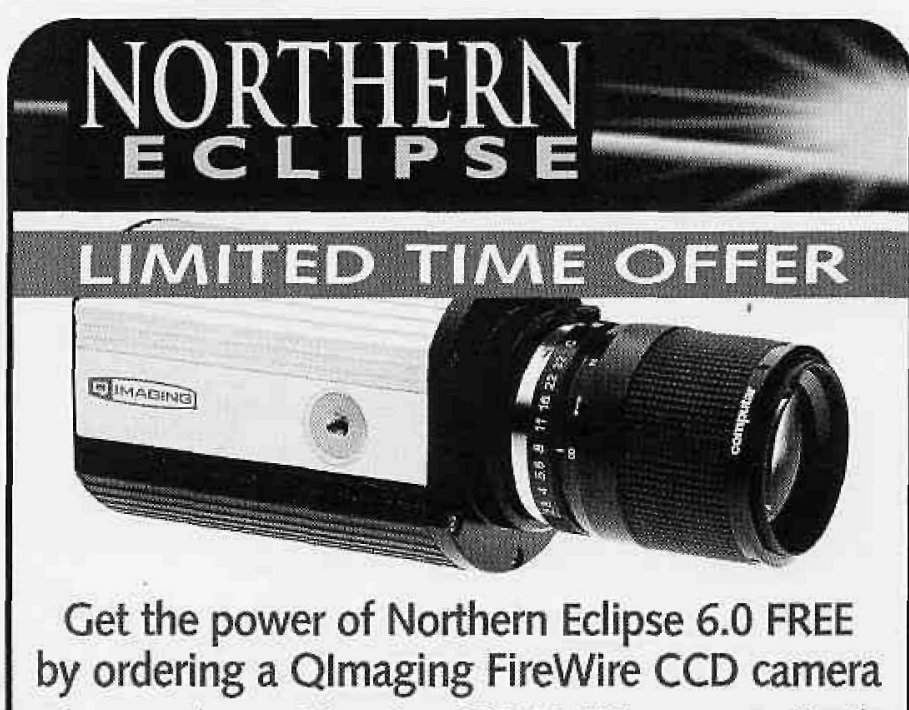

When you buy a QImaging CCD FireWire camera directly from us at the manufacturer's suggested list price, we will include, for a limited time, one FREE Northern Eclipse 6.0 application of your choice. Applications such as Brightness/ Time, Time-Lapse, Image Merging, Fast Capture to Ram, and even De-convolution are available. See the On Sale page of our web site for a full list of applications available with this offer.

\section{www.empix.com}

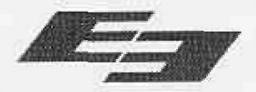

E M P I X 


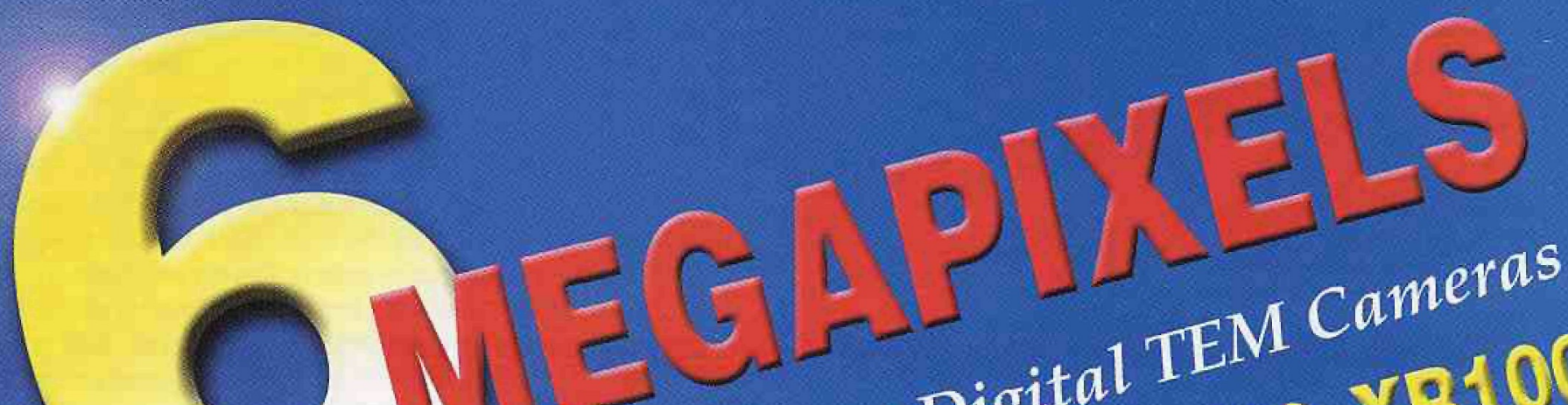
High Definition Digital $\times R 60$ \& XR100

TETEQRATION

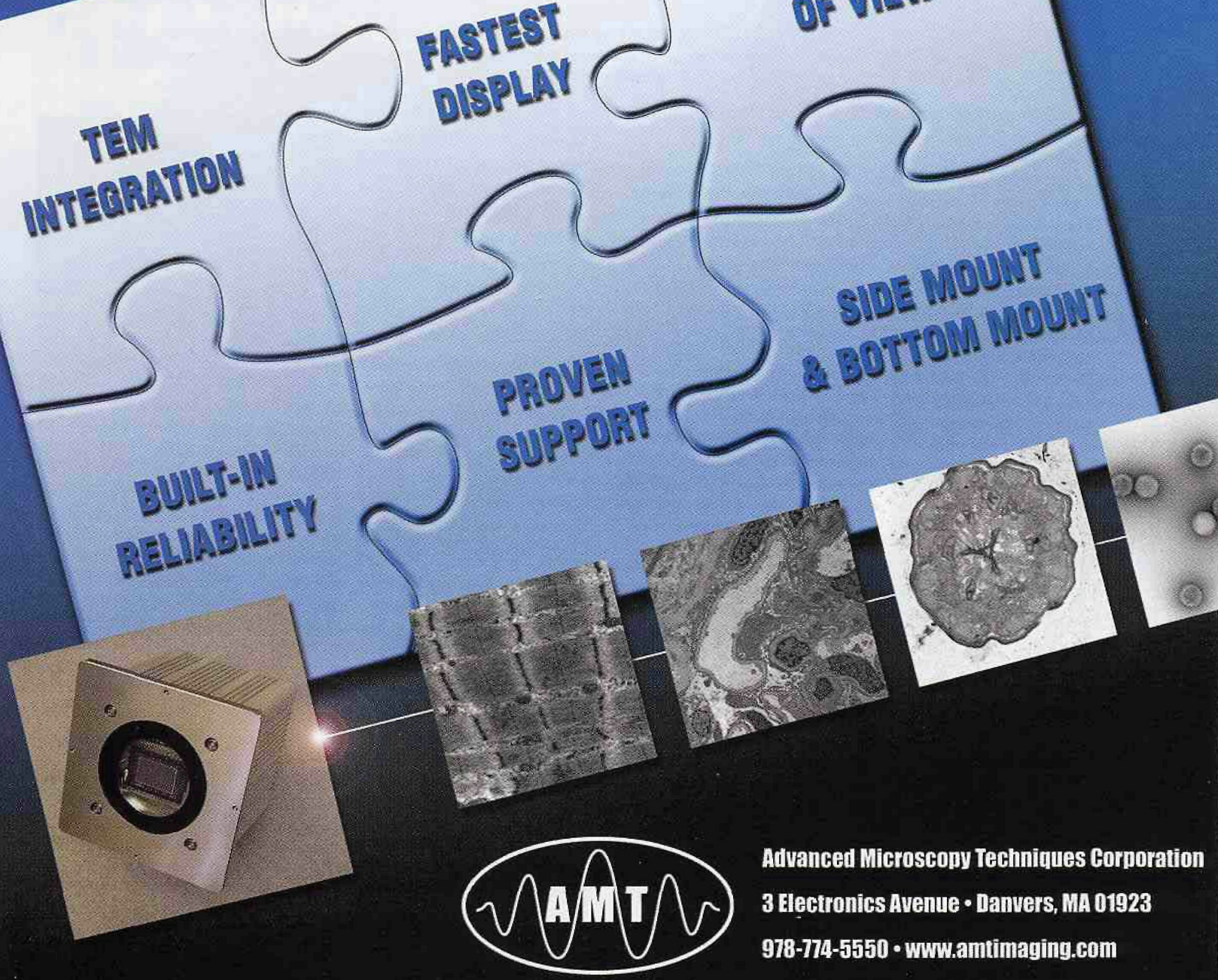

Advanced Mieroseopy Techniques Corporation a bortolin Mo of VIEW 